\title{
Spectral Line Variations of Symbiotic Stars EG And, AG Dra, and BX Mon and Its Interpretation
}

\author{
Tae Seog Yoon ${ }^{1}$, Soo Hyun Kim ${ }^{1}$, Hyeonwoo Moon ${ }^{1}$, \\ Kyu-Seob Kim ${ }^{1}$, and Hyungil $\mathrm{Oh}^{1,2}$ \\ ${ }^{1}$ Department of Astronomy \& Atmospheric Sciences, Kyungpook National University, \\ Daegu 702-701, South Korea \\ email: yoonts@knu.ac.kr \\ ${ }^{2}$ Korea Astronomy and Space Science Institute, Daejeon 305-348, South Korea
}

\begin{abstract}
We present some results obtained by high resolution spectroscopic observations for symbiotic stars EG And, AG Dra, and BX Mon in recent years which were performed with 1.8-m reflector and echelle spectrograph BOES at Bohyunsan Optical Astronomy Observatory, Youngcheon, South Korea. The variations of $\mathrm{H} \alpha$ emission line during a night and the variations of $\mathrm{H}$ Balmer lines and He I emission lines among several analyzed lines over months and years are shown and discussed.
\end{abstract}

Keywords. Symbiotic stars: EG And, AG Dra, BX Mon, Spectral line variations

In order to investigate any variations of spectral lines for symbiotic stars two methods were adopted. One is to find spectral line variations in a very short time scale such as flickering phenomena by time-series exposures over an observing night. Another is to find line variations in the time scales of months or years.

Through overnight monitoring spectral line variation for a night is detected only for AG Dra among selected symbiotic stars and only from the last frame of Feb. 24, 2008. That frame was taken just before the morning twilight. Overlapped time-series $\mathrm{H} \alpha$ spectra of AG Dra on the night are shown in Figure 1. While Dobrzycka et al. (1996) and Sokoloski et al. (2001) commented possibilities of flickering phenomena for EG And, AG Dra, and BX Mon, we are not quite successful so far in finding the very short-time variation in spectral lines by overnight monitoring.

Appearance and disappearance of double-peak shape of $\mathrm{H} \alpha$ and $\mathrm{H} \beta$ lines are shown for EG And and AG Dra over years, and more prominent at $\mathrm{H} \alpha$ line. Shift of doublepeak $\mathrm{H} \alpha$ line and its variation in line intensity and line width are detected for BX Mon. $\mathrm{H} \alpha$ line variation of EG And is shown in Figure 2. Variations of He I $\lambda 5875, \lambda 6678, \lambda 7065$ lines for AG Dra are shown in Figure 3. The variation pattern of He I $\lambda 5875$ and $\lambda 7065$ lines looks similar, but that of $\lambda 6678$ line is different.

Acknowledgments: This research was supported by Basic Science Research Program through the National Research Foundation of Korea (NRF) funded by the Ministry of Education, Science and Technology (No. 2010-0023430, No. 2011-0026126, \& No. 2012R1A1A2006759). 


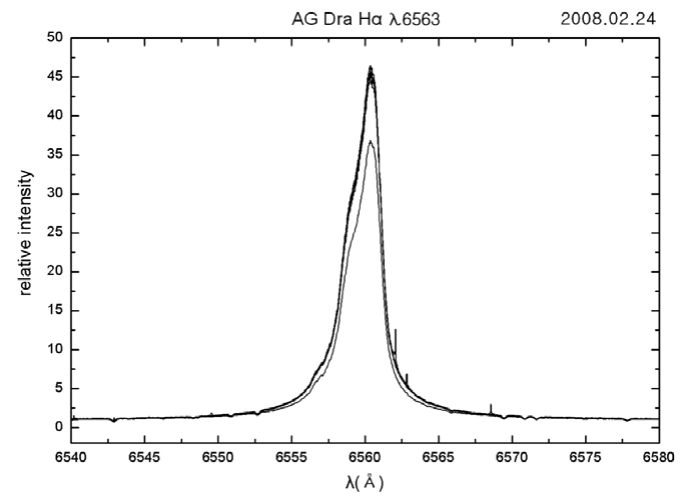

Figure 1. Overlapped AG Dra H $\alpha$ spectra of Feb. 24, 2008

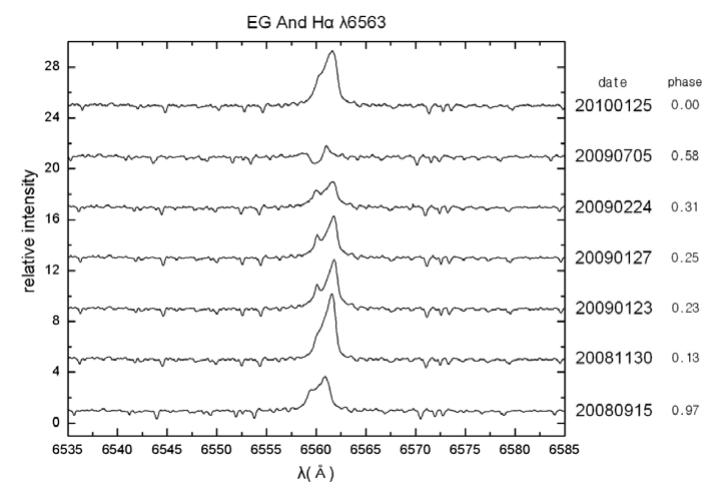

Figure 2. $\mathrm{H} \alpha$ lines of EG And observed from Sept. 2008 to Jan. 2010

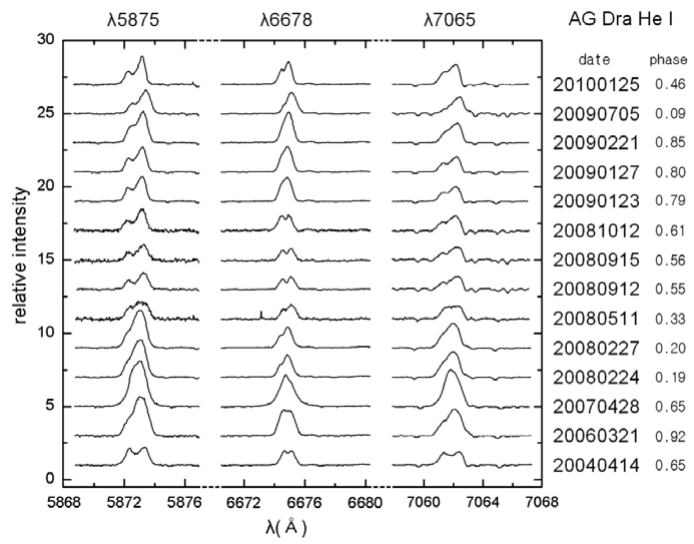

Figure 3. Variations of He I $\lambda 5875, \lambda 6678, \lambda 7065$ lines of AG Dra

\section{References}

Dobrzycka, D., Kenyon, S. J., \& Milone, A. A. E. 1996, AJ, 111, 414

Sokoloski, J. L., Bildsten, L., \& Ho, W. C. G. 2001, MNRAS, 326, 553 